\title{
SIMULATION METHOD FOR DROPPER DYNAMIC LOAD CONSIDERING HORIZONTAL VIBRATION BEHAVIOUR
}

\author{
Zhang, J. W.; Wu, J. Q.; Chen, W. R.; Guan, J. F.; Zhong, Y. \& Xu, K. J.\# \\ School of Electrical Engineering, Southwest Jiaotong University, Chengdu 611756, China \\ E-Mail: kjxu@ @wjtu.edu.cn ( ${ }^{\#}$ Corresponding author)
}

\begin{abstract}
Dropper is the key component of catenary in high-speed railways. The prediction of the fatigue life of dropper is accomplished using alternating load characteristics. Simulation method for obtaining the dynamic internal load of the dropper in previous studies was insufficient for accurate requirements in predicting dropper life. According to realistic working conditions in the simulation environment, this study proposed a simulation method considering horizontal vibration behaviour for the dropper. The horizontal vibration phenomenon of a dropper was determined on the basis of observing and measuring a real high-speed railway. Moreover, a test for the reappearance of horizontal vibration in the laboratory was performed. The dropper was modelled with multibody dynamics. Finally, the horizontal vibration behaviour of the dropper on-site was reproduced in a laboratory test and computer simulation. Results demonstrated that the coefficient of association between the virtual sensor load in a simulation environment and the real sensor load in a laboratory test is 0.9578 . The proposed method provides an accurate approach to simulating the load of the droppers under real working conditions. (Received in July 2019, accepted in November 2019. This paper was with the authors 1 month for 2 revisions.)
\end{abstract}

Key Words: High-Speed Railway, Catenary, Dropper, Horizontal Vibration, Simulation Model

\section{INTRODUCTION}

Pantograph-catenary system is an important subsystem in high-speed railways. The catenary transmits electrical energy to a high-speed train through the pantographs installed on the roof of the train while moving at high speed. Droppers are the connections between the contact wire and the messenger wire. With different locations and lengths, the droppers place the contact wire in a specific location above the rails [1]. Typically, both ends of a dropper are electrically connected by current loops. Then, the main line of a dropper bears the main mechanical loads.

During the long-term vibration caused by the dynamic action of the pantograph-catenary system, the droppers may fracture due to fatigue, especially at the end of the main line. Numerous droppers are used in the traction power supply system in high-speed railways. More than 7 million sets of droppers in $29,000 \mathrm{~km}$ of high-speed railways in China are in service as of the end of 2018. A failure of the pantograph-catenary system may occur once a dropper fails. This incident may seriously affect the safe operation of the high-speed railway. Moreover, frequent failures of the pantograph-catenary system may significantly increase maintenance and repair work.

From case studies, the fatigue modes of droppers that have been mastered are mostly due to metal fatigue [2]. The fatigue life of a dropper under real working conditions depends on the load-life characteristics of the dropper and the actual dynamic load in the dropper. The load-life characteristics of the dropper can be obtained by subjecting the dropper to a fatigue test. According to the theory of damage accumulation in metal fatigue, the magnitude and number of alternating loads on metal specimens play a decisive role in the accumulation of fatigue damage [3]. However, to measure the dynamic load in an operating high-speed railway by installing a force sensor on a dropper is difficult due to three reasons. First, installing unreliable sensors may threaten the safety of high-speed railways. Second, installing the sensor may affect the original situation of the droppers. Third, obtaining accurate data in 
high-voltage and large-current situations is hard. Therefore, computer simulation becomes an ideal and highly efficient means of obtaining the dynamic load in the dropper.

Obtaining the dynamic load of droppers in the pantograph-catenary system through computer simulation has been studied [4]. However, the simulation process ignores the horizontal vibration behaviour of the droppers. To study the fatigue lives of the droppers, obtaining the load inside the dropper is an essential prerequisite. Thus, a simulation method considering horizontal vibration behaviour must be considered.

Based on the abovementioned analysis, this study takes the horizontal phenomenon of the droppers as the breakthrough point. Then, a simulation method based on multibody dynamics for the horizontal behaviour of a dropper is set up.

\section{STATE OF THE ART}

In the analysis and prediction of dropper fatigue lives, Chen et al. [5] carried out a simulated calculation based on laboratory conditions for the relationships between the frequency, amplitude, and force of the dropper in the fatigue tests. However, only the vertical vibration was studied. Yang [6] investigated the characteristics of the fatigue life of droppers through a test device driven from the upper end of a dropper. During the testing procedure, the horizontal vibration of the droppers was not mentioned. Peng et al. [7] used the test method driven at the upper end when studying the effects of frequency and amplitude of the test device. Liu et al. [8] conducted the fatigue tests of droppers, but only the vertical load of the dropper was considered.

In the mechanism of the fatigue fracture of the droppers, Yang [9] mentioned the influence of reciprocating a dropper when analysing the mechanism of the fracture in the droppers. However, the bending behaviour was not horizontal vibration behaviour exactly. Numerical simulation is often used to calculate the forces in the structures [10]. Tan et al. [11] studied the mechanism of the dropper fatigue phenomenon from the aspect of the fretting theory. Jiang et al. [12] focused on the mechanism of the fatigue fracture in a dropper. When the microphenomenon was studied, macroscopical vibration behaviours of the dropper were ignored.

The model of the dropper is the basis of the pantograph-catenary simulation system. Dropper models were mentioned in the dynamic simulation of pantograph-catenary. Verifying the correctness of the simulation through experiments is the basic logic [13]. Finite element methods are often used to analyse and optimize structures [14]. Guan and $\mathrm{Wu}$ [15] established the dynamic simulation model of the pantograph-catenary system. This model was verified through on-site tests. The dropper model was suitable for the whole system. However, the model was insufficient for simulating the horizontal vibration behaviour. Zhao et al. [16] and Qi et al. [4] used the same method to establish the system model, but only the vertical vibration was calculated. Benet et al. [17] and Pombo and Ambrosio [18] studied a simulation model of the pantograph-catenary system, but the dropper was simplified into a finite element calculation unit. Gregori et al. [19] and Massat et al. [20] considered the droppers similar to elastic traction bars, which could not vibrate horizontally. Cho [21] simplified a dropper into a spring and a damping force (i.e., a mass-spring model), which was only allowed to vibrate vertically. Bruni et al. [22] modelled the droppers as bilinear elements with zero tension in their slackening. Similarly, Vo Van et al. [23] modelled the droppers as bars with nonlinear stiffness to represent the loss of stiffness in compression. Only the vertical load required attention. Gregori et al. [24] presented a fast simulation method of the pantograph-catenary dynamic interaction. The dropper model included in the method was inadequate to support the studies of the horizontal vibration of droppers. 
The simplified dropper model in the literature only considered the longitudinal movement of the droppers. The oversimplified model of the dropper was insufficient for describing the true vibration process of the dropper and its relationship with the internal load accurately. The present study proposes a method for simulating the dropper dynamic load in high-speed railway catenary. The horizontal vibration behaviour of the dropper is considered by establishing a simulation model of a dropper based on multibody dynamics. Moreover, the model can be verified through laboratory and on-site tests. The method provides a new step for approaching the real working conditions of the droppers.

The remainder of this study is organized as follows. Section 3 introduces the capture of the dropper vibration in the real high-speed railway situation. A device is built for simulating the horizontal vibration behaviour of droppers. Simultaneously, the dropper model considering horizontal vibration is proposed. Section 4 discusses the applicability of the method in accordance with the results from on-site measurement, laboratory test, and computer simulation. Section 5 summarizes the conclusions drawn from this study.

\section{METHODOLOGY}

\subsection{On-site measurement in real high-speed railway situation}

In the catenary system, the dropper connects the contact wire and the messenger wire. In the static state, the dropper receives the gravity of the contact wire nearby. Under a maintained axial tension, the dropper remains motionless in the tension mode. However, the contact wire rises sharply and rapidly when the pantograph passes at high speed. At this time, the messenger wire rises at a proper velocity and has a limited rising range. The internal tension of the dropper decreases rapidly, and the slack of the dropper appears. After the pantograph passes, the contact wire falls, and the dropper tightens again. In this process, the dropper becomes unstable and horizontal vibration progresses.

To verify the horizontal vibration process, this study performs the measurement through the noncontact method at an actual high-speed railway site. The target dropper is selected from a $350 \mathrm{~km} / \mathrm{h}$ high-speed railway catenary in China. A high-speed camera is set up near the railway and aims at the target dropper, as shown in Fig. 1a. The frequency of the image capture is $2,000 \mathrm{~Hz}$. The images are stored continuously on a computer. The position data of the contact wire, messenger wire, and the dropper are extracted from the images through an edge detector method. The continuous position data are used to construct the vibration curves. Every edge between the white and black regions can be detected, as illustrated in Fig. $1 \mathrm{~b}, 1 \mathrm{c}$, and $1 \mathrm{~d}$. The red arrows are the detected vibration positions and directions.

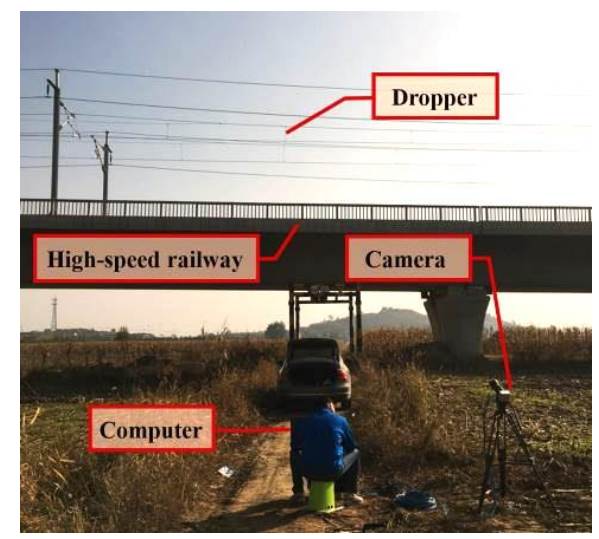

a)

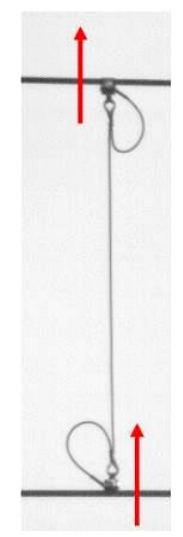

b)

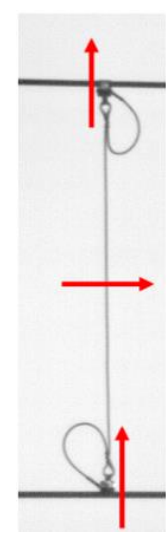

c)

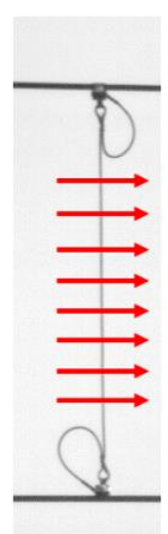

d)

Figure 1: Measurement devices and principles: a) Measurement devices in an actual high-speed railway scene; b), c), and d) Cases for the edge detection of positions and directions. 


\subsection{Method and device in the laboratory test}

To reproduce the actual vibration process of the dropper in the laboratory, a dropper vibration test method is proposed, as depicted in Fig. 2 a. The upper end of the dropper is connected to a spring with a stiffness of $k_{1}$ using a set of fasteners and a force sensor. The mass above the force sensor is $m_{s 1}$. The mass below the force sensor is $m_{s 2}$. The fasteners and the force sensor are connected rigidly. The lower end of the dropper is connected to the first load with a mass of $m_{c 1}$. The second load with a mass of $m_{c 2}$ is connected to the first load using a spring with a stiffness of $k_{2}$. The purpose of setting the second load is to extend the time in which the dropper tightens. The position of the first load represents the position of the contact wire. Except for the dropper, the loads, springs, and fasteners are limited to only moving in the vertical direction and cannot be rotated. The test equipment established in the laboratory is demonstrated in Fig. 2 b.

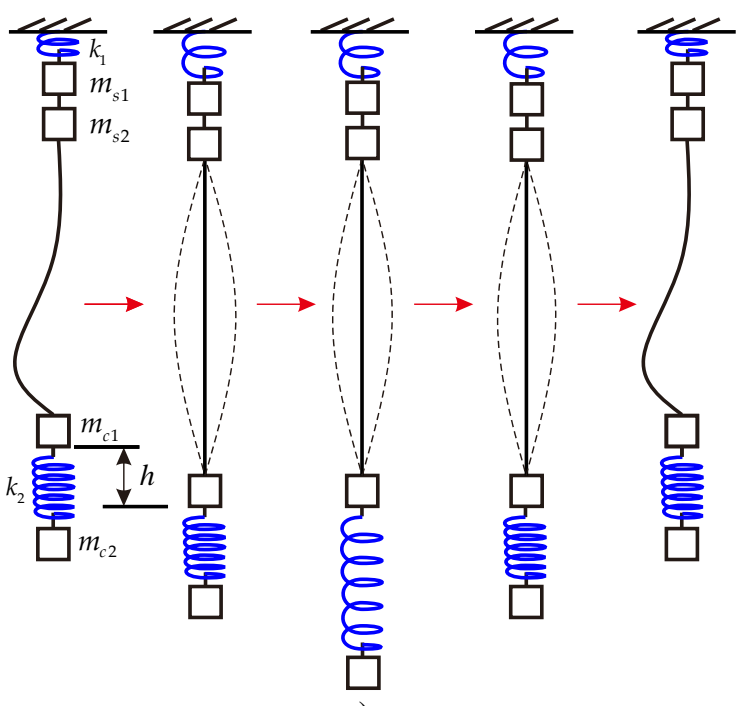

a)

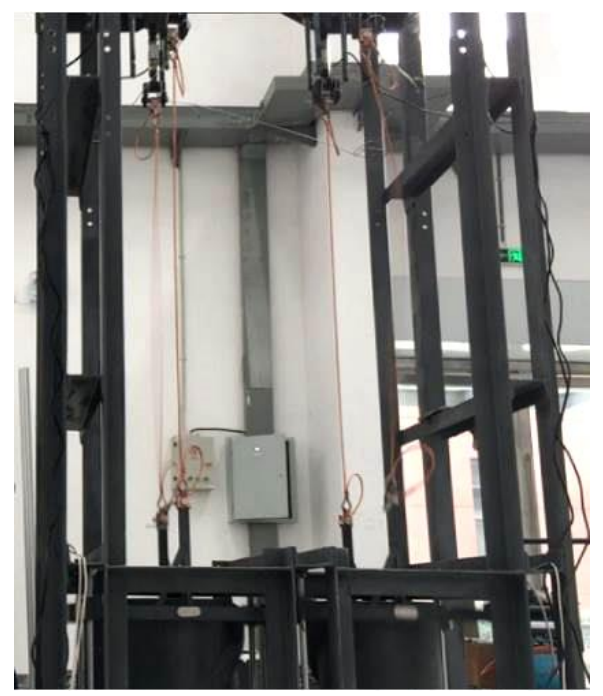

b)

Figure 2: Method and device for testing the dropper vibration: a) Method for testing the dropper vibration; b) Device for testing the dropper vibration.

Table I: Key parameters of the laboratory test.

\begin{tabular}{|c|c|c|}
\hline Variable & Unit & Value \\
\hline$k_{1}$ & $\mathrm{~N} / \mathrm{m}$ & 20,177 \\
\hline$k_{2}$ & $\mathrm{~N} / \mathrm{m}$ & 998 \\
\hline$m_{s 1}$ & $\mathrm{~kg}$ & 0.49 \\
\hline$m_{s 2}$ & $\mathrm{~kg}$ & 0.55 \\
\hline$m_{c 1}$ & $\mathrm{~kg}$ & 3.5 \\
\hline$m_{c 2}$ & $\mathrm{~kg}$ & 5.0 \\
\hline$h$ & $\mathrm{~mm}$ & 70 \\
\hline$L$ & $\mathrm{~mm}$ & 1,070 \\
\hline
\end{tabular}

The movement process in the test is described as follows. First, $m_{c 1}$ and $m_{c 2}$ are lifted to the height of $h$ to move the dropper into the loosening status. Second, $m_{c 1}$ and $m_{c 2}$ fall freely. When the dropper is tightened, $m_{c 1}$ stops falling naturally. Given its inertia, $m_{c 2}$ continues to fall and stretches the spring of $k_{2}$ until the lowest point and returns to the original position. During the test, the upper end of the dropper (which represents the messenger wire), the lower end of the dropper (which signifies the contact wire), and the horizontal vibration of the dropper are measured through the noncontact measurement method. In addition, the force measurement data of the force sensor are recorded. The key parameters of the test are listed in Table I. 


\subsection{Dynamic simulation model of the dropper}

According to the current study on the dropper simulations mentioned above, the method for simplifying the dropper into spring and damping cannot show the horizontal movement of the dropper. Actually, the model of the dropper must consider the large deformation behaviour of the dropper main line. The structure of a dropper is complex. This structure consists of $7 \times 7$ spiralled wires. One option to represent this structure is an entity simulation model; however, the calculations for this approach are difficult and complex [25]. An alternative option has been reported in the literature, in which a continuous model in the simulation environment can be divided into multiple sections [26].

The present study adopts the transfer matrix method of the multibody system for modelling a dropper. A dropper with a length of $L$ is divided into $n$ sections. Each section is a rigid body with a mass of $m_{0}$ and a length of $l_{0}$. This section is calculated using the line density of the real dropper. A virtual and massless planar torsion spring with a torsional stiffness of $k_{T}$ is located between two neighbouring sections. The current line is neglected in this study to facilitate modelling. The model is exhibited in Fig. 3.

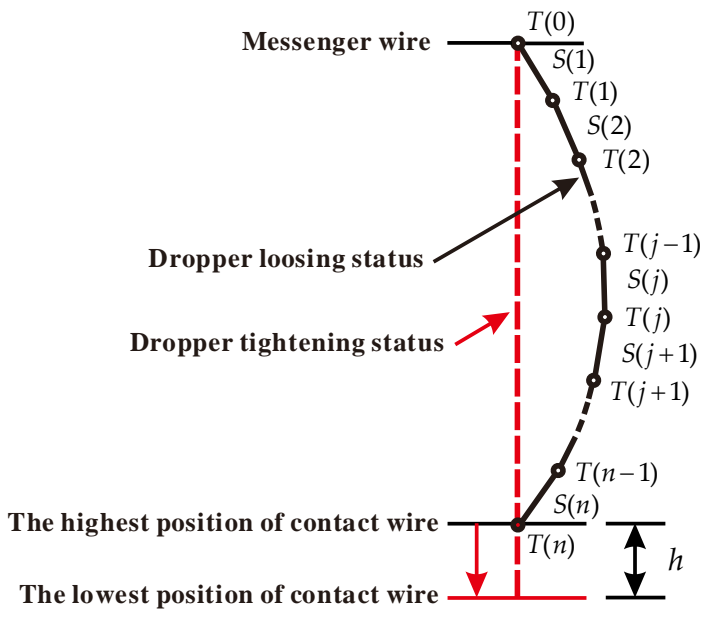

a)

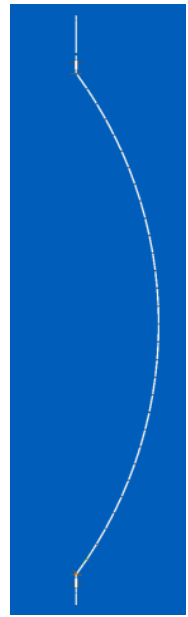

b)

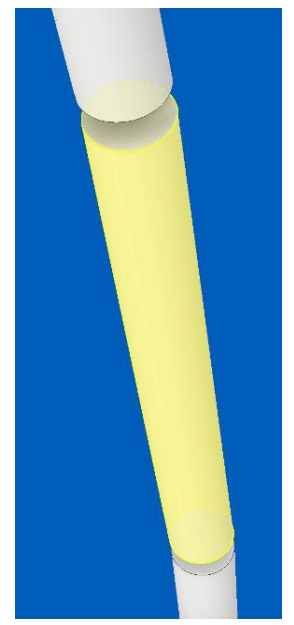

c)

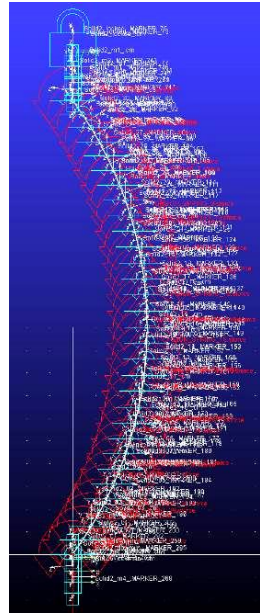

d)

Figure 3: Simulation model of a dropper: a) Theoretical model; b) Whole model; c) Rigid body in the model; d) Constraints of the model.

In Fig. 3 a, $S(1), S(2), \ldots, S(n)$ are the rigid bodies that are articulated, and the transfer matrixes are $\mathbf{U}_{S(1)}, \mathbf{U}_{S(2)}, \ldots, \mathbf{U}_{S(n)}$, respectively. $T(1), T(2), \ldots, T(n)$ are the planar torsion springs, and the transfer matrixes are $\mathbf{U}_{T(1)}, \mathbf{U}_{T(2)}, \ldots, \mathbf{U}_{T(n)}$, correspondingly. Rui et al. conducted many works in the multibody system through the transfer matrix method. The transfer matrix method was clearly verified [26]. Through the transfer matrix method, the one-end input and output rigid body state vectors are:

$$
\mathbf{z}=\left[x, y, \theta, m, q_{x}, q_{y}, 1\right]^{\mathrm{T}}
$$

$x, y$ is the position on the model, $\theta$ is the angle of the rigid body, $m$ is the mass of the rigid body, and $q_{x}$ and $q_{y}$ are the internal forces.

The transfer equations in a dropper main line are:

$$
\begin{gathered}
\mathbf{z}_{T(j), S(j)}=\mathbf{U}_{S(j)} \mathbf{z}_{S(j), T(j-1)} \\
\mathbf{z}_{S(j+1), T(j)}=\mathbf{U}_{T(j)} \mathbf{z}_{T(j), S(j)} \\
\mathbf{z}_{T(j+1), S(j+1)}=\mathbf{U}_{S(j+1)} \mathbf{z}_{S(j+1), T(j)}
\end{gathered}
$$


The transfer equations of the upper end of the dropper are:

$$
\begin{gathered}
\mathbf{z}_{S(1), T(0)}=\mathbf{U}_{T(0)} \mathbf{z}_{T(0)} \\
\mathbf{z}_{T(1), S(1)}=\mathbf{U}_{S(1)} \mathbf{z}_{S(1), T(0)}
\end{gathered}
$$

The transfer equations of the lower end of the dropper are:

$$
\begin{gathered}
\mathbf{z}_{T(n), S(n)}=\mathbf{U}_{S(n)} \mathbf{z}_{S(n), T(n-1)} \\
\mathbf{z}_{T(n)}=\mathbf{U}_{T(n)} \mathbf{z}_{T(n), S(n)}
\end{gathered}
$$

The total transfer equation can be assembled as:

$$
\mathbf{z}_{T(n)}=\mathbf{U}_{T(n)} \mathbf{U}_{S(n)} \mathbf{U}_{T(n-1)} \ldots \mathbf{U}_{S(j+1)} \mathbf{U}_{T(j)} \mathbf{U}_{S(j)} \ldots \mathbf{U}_{S(2)} \mathbf{U}_{T(1)} \mathbf{U}_{S(1)} \mathbf{U}_{T(0)} \mathbf{z}_{T(0)}
$$

A simulation model of the dropper vibration is established in the ADAMS 2016 software of MSC Software Corporation ${ }^{\circledR}$. The initial state of the model is the dropper loosening status displayed in Fig. 3 a. The simulation model in the software is illustrated in Fig. 3 b, 3 c, and 3 d.

In the simulation model, $k_{1}, m_{s 1}, m_{s 2}, h$, and $L$ have the same values as the laboratory test parameters. The simulation parameters are listed in Table II.

Table II: Key parameters of the simulation.

\begin{tabular}{|c|c|c|}
\hline Variable & Unit & Value \\
\hline$k_{1}$ & $\mathrm{~N} / \mathrm{m}$ & 20,177 \\
\hline$m_{s 1}$ & $\mathrm{~kg}$ & 0.49 \\
\hline$m_{s 2}$ & $\mathrm{~kg}$ & 0.55 \\
\hline$h$ & $\mathrm{~mm}$ & 70 \\
\hline$n$ & $/$ & 30 \\
\hline$l_{0}$ & $\mathrm{~mm}$ & 35.7 \\
\hline$m_{0}$ & $\mathrm{~g}$ & 3.16 \\
\hline$k_{T}$ & $\mathrm{Nm} / \mathrm{deg}$ & 0.01112 \\
\hline$L$ & $\mathrm{~mm}$ & 1,070 \\
\hline
\end{tabular}

\section{RESULTS ANALYSIS AND DISCUSSION}

\subsection{Horizontal vibration of the droppers in real high-speed railway situation}

When a typical high-speed train with two working pantographs passes the measurement location at approximately $350 \mathrm{~km} / \mathrm{h}$, the images are captured, as shown in Fig. 4 a.

The process of the horizontal vibration of the dropper is analysed qualitatively and theoretically, as depicted in Fig. 4 b. The process consists of seven statuses. Status I is the posture in the static state of the catenary. The dropper is tightened by the gravity of the adjacent contact wire and remains motionless. In Status II, a passing pantograph leads to the narrowing of the distance between the contact wire and the messenger wire. Considering that the dropper cannot be compressed directly, it moves to one side and deviates from the static position. After the pantograph passes, the contact wire drops, and the dropper moves back to the equilibrium position. Simultaneously, $d_{0}$ is reduced gradually, and the horizontal movement speed of the dropper main line increases gradually. From this time, $F_{\text {down }}$ begins to affect the system, and the dropper begins to tighten. In Status III, the horizontal movement speed of the dropper $v_{0}$ reaches a maximum value. Given inertia, the dropper continues to move away from the equilibrium position until it deviates from the equilibrium position at $d_{1}$ and stops, as shown in Status IV. Afterward, the dropper starts to move in the opposite direction. When the dropper line moves to Status V, it produces the reverse maximum speed and continues to move to the position away from the equilibrium position to $d_{2}$ in Status VI. Status VII reflects the dropper line returning to the equilibrium position. 


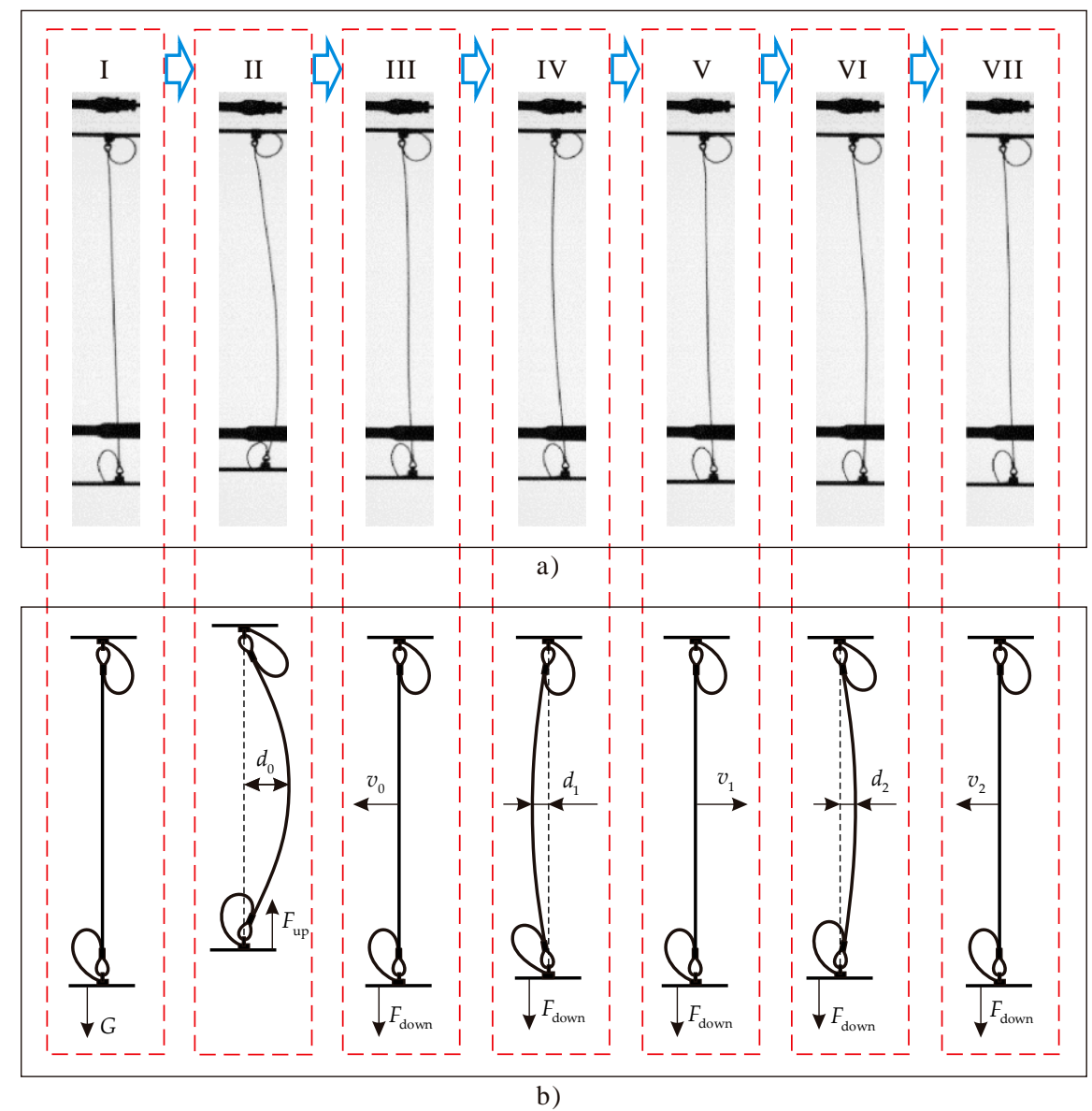

Figure 4: Phenomenon and analysis of the deformation and vibration behaviour of a dropper during a high-speed pantograph passing: a) Images captured at a high-speed railway site; b) Pictures illustrating the theoretical process of the dropper vibration behaviour.

In quantitative analysis, the dropper vibration curves are detected by the edge-detecting position presented, as plotted in Fig. $1 \mathrm{~b}$. The vibration displacements of the contact wire and the messenger wire are illustrated in Fig. 5.

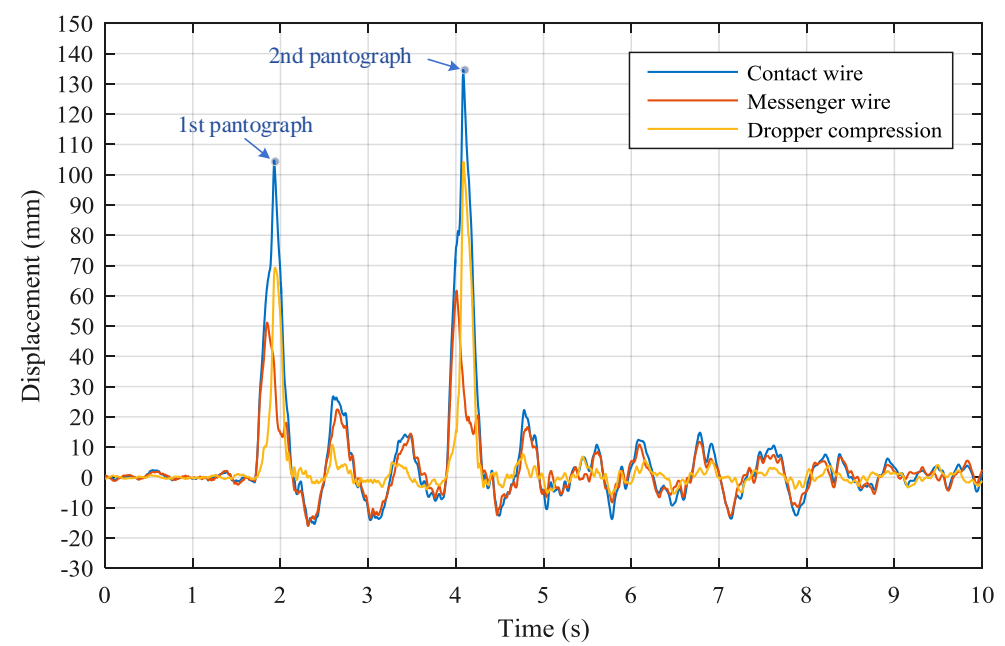

Figure 5: Vibration displacements of contact wire and messenger wire when a typical high-speed train with two working pantographs passes by.

Fig. 5 shows that the contact wire is lifted obviously when the two pantographs are passing. The dropper compress is defined as the difference between the displacements of the 
contact wire and messenger wire. It represents the looseness of the dropper. When a pantograph is passing, the dropper compress displacement reaches a peak value.

For a clear understanding of the loosening and tightening details of the dropper, a magnified version of Fig. 5, between 1.5 and 2.8 s, is presented in Fig. $6 \mathrm{a}$. An additional curve of the dropper horizontal vibration displacement is included in the figure.

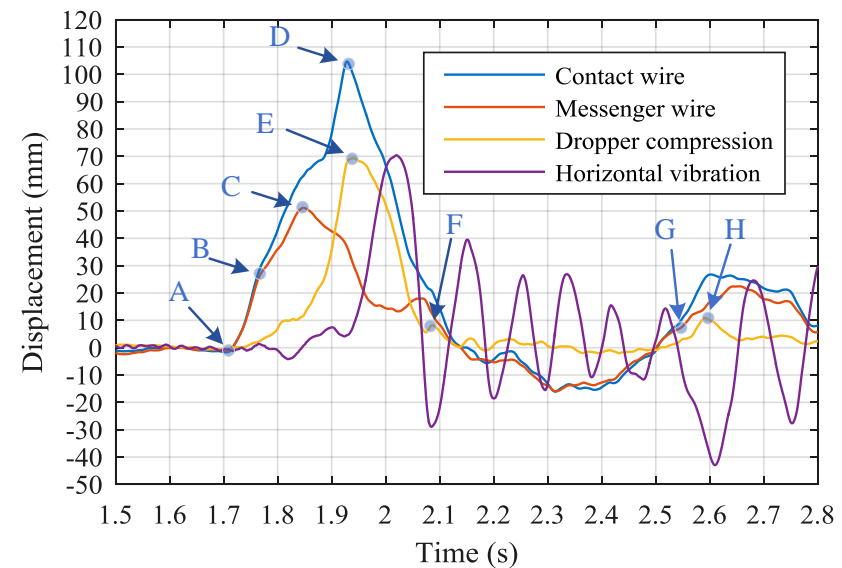

a)

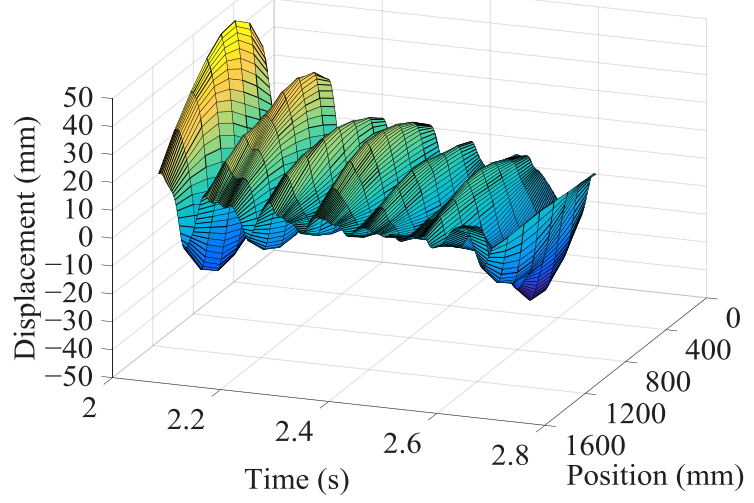

b)

Figure 6: Results under real working conditions: a) Magnified version of Fig. 5 with an additional curve of the dropper horizontal vibration displacement; b) 3D curve of the dropper horizontal vibration under real working conditions.

The detected positions and directions of the curves in Fig. 6 a come from Fig. 1 c. In Fig. $6 \mathrm{a}$, at first, the pantograph is gradually approaching to the target dropper initially. The contact wire slowly rises from Point A. Given the wire tension, the messenger wire rises synchronously. The dropper keeps tightening until Point B. At Point B, the rising speed of the messenger wire begins to fall behind the contact wire. The movement of the contact wire becomes inconsistent with the messenger wire, thereby resulting in the dropper starting to loosen. The lifting force caused by the tension of the messenger wire slightly reduces. The messenger wire stops moving upward at Point $\mathrm{C}$ and starts to vibrate downward freely. During this procedure, the contact wire is moving upward continuously. This movement is caused by the approaching pantograph. The dropper compress value continues to increase. At Point $\mathrm{D}$, the pantograph is right under the target dropper, and the position of the contact wire reaches the highest point. Nearly at the same time, the maximum value of the dropper compress appears at Point E. The contact wire begins to move downward after the pantograph passes. The dropper compression gradually decreases. This phenomenon represents the dropper's conversion from the loosening status to the tightening status. This effect occurs until the distance between the messenger wire and the contact wire is approximately equal to the length of the dropper. The messenger wire starts to move downward in response to the force from the contact wire through the tightened dropper at Point F. Then, the dropper keeps tightening until Point G. During the time between Points F and G, the dropper vibrates horizontally, as reflected in the curve of horizontal vibration plotted in Fig. 6 a.

Eight recognition positions shown in Fig. $1 \mathrm{~d}$ are set on the images to describe the horizontal vibration of the dropper clearly. Then, a 3D curve of the dropper horizontal vibration is obtained, as illustrated in Fig. 6 b.

In Fig. 6 b, the position axis refers to the location on the dropper main line, which starts from one end. The horizontal vibration phenomenon occurs in the dropper main line. Together with the results in Fig. 6 a, a series of phenomena is revealed. Initially, the unsynchronized movement of the contact wire and messenger wire loosens the dropper. Then, the dropper vibrates horizontally during the subsequent tightening procedure. 


\subsection{Reappearance of the horizontal vibration behaviour of a dropper in the laboratory}

The dropper vibration test is performed using the device built in the laboratory. The test results are demonstrated in Fig. 7 a. The dropper compression displacement is the difference of displacements between the upper and lower ends of the dropper. The dropper is tightened from Time A and remains tight until Time B. The compression amount of the dropper fluctuates at approximately zero, thus indicating that the dropper is in a tight state. As the method of measurements under real working conditions in Fig. 1 d, a 3D curve of the dropper horizontal vibration is obtained and depicted in Fig. 7 b.

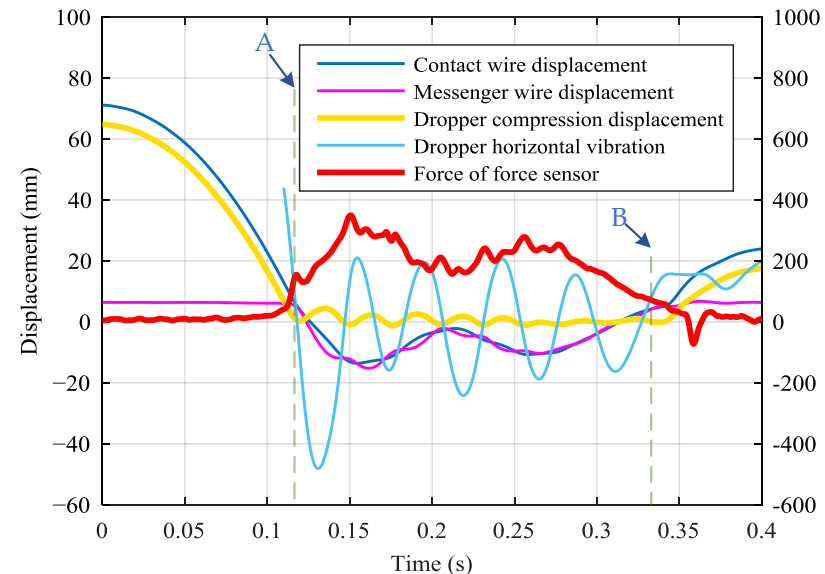

a)

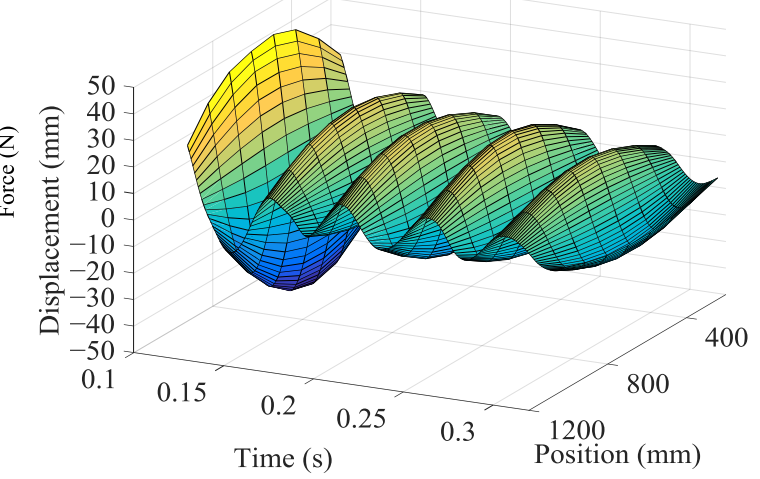

b)

Figure 7: Results in the laboratory: a) Displacements and force measurement results of the test in the laboratory; b) 3D curve of the dropper horizontal vibration in the laboratory test.

Fig. $7 \mathrm{~b}$ shows that the dropper is vibrating horizontally. This movement is similar to the process under real working conditions. Dropper horizontal vibration is simulated in the laboratory.

\subsection{Dynamic simulation results}

During the simulation, the outer situation must be the same as in the laboratory test. In the laboratory test, the active movement of the contact wire is the power of the whole test system. Then, the displacement curve of the contact wire in the laboratory test is used as the driving curve of the contact wire in the simulation environment.

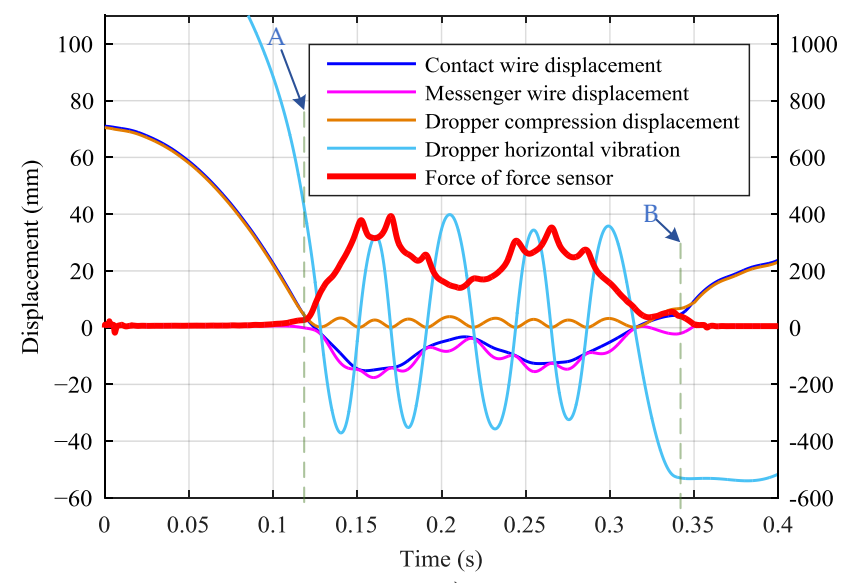

a)

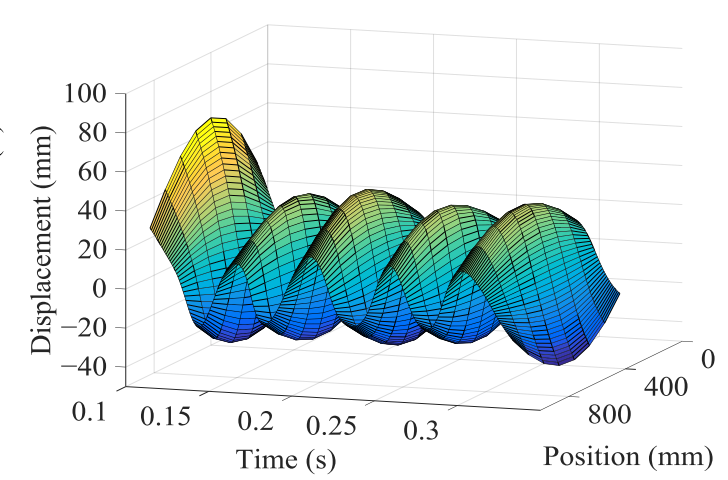

b)

Figure 8: Results of simulation: a) Displacements and force measurement results of the simulation; b) $3 \mathrm{D}$ curve of the dropper horizontal vibration in the simulation. 
The results in the simulation environment can be obtained directly. To correspond to the results of the laboratory test, only the displacement of the contact wire and messenger wire, dropper horizontal vibration, and the force of the virtual force sensor are obtained, as exhibited in Fig. 8 a.

A 3D curve of the dropper horizontal vibration obtained in the simulation environment is illustrated in Fig. $8 \mathrm{~b}$ to facilitate the comparison with the results from the laboratory test and actual measurement.

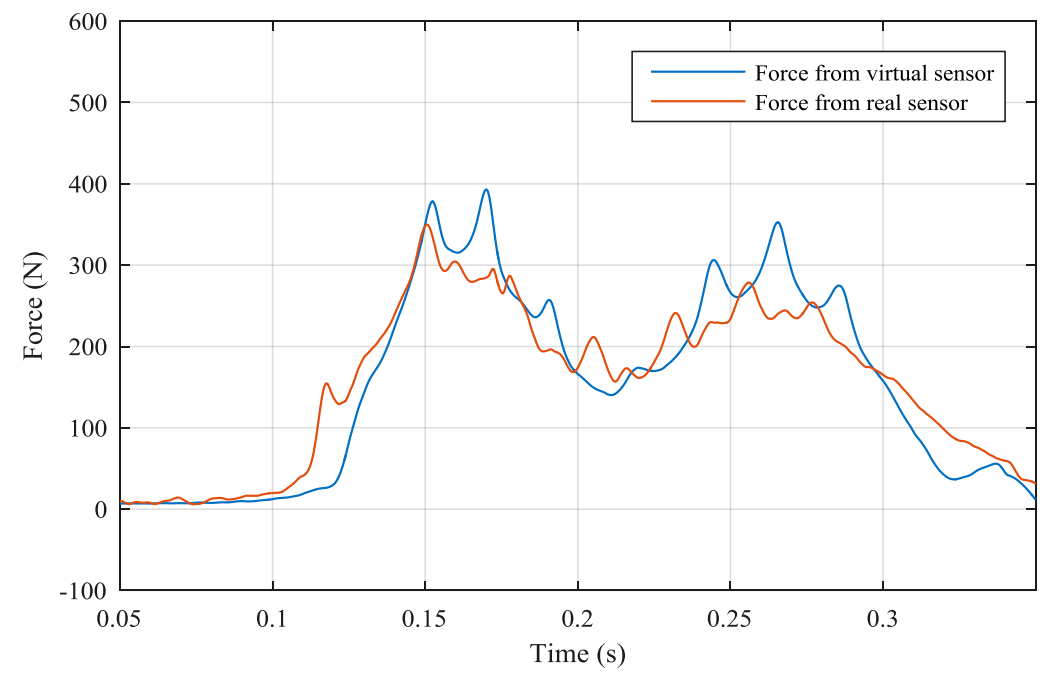

Figure 9: Comparison between the sensor forces in the real and the virtual sensors.

Fig. 9 reflects the combination of the force of the simulated (virtual) force sensor depicted in Fig. $8 \mathrm{a}$ and the real force sensor displayed in Fig. 7 a.

The calculation presented in Fig. 9 shows that the coefficient of association reached 0.9578. Simultaneously, the curves in Figs. 6, 7, and 8 show that the droppers in the real working conditions, laboratory test and in the simulation have the same features. First, the dropper vibrates horizontally. Second, the amplitudes of the horizontal vibrations are large and cannot be ignored. These amplitudes can be considered large deformations. Finally, the dropper vibrates left and right several times.

\section{CONCLUSIONS}

A dropper dynamic simulation model was built by considering the horizontal vibration behaviour to establish a computer simulation method of dropper vibration for a close reappearance under real working conditions. The horizontal vibration behaviour of the dropper in high-speed railway catenary is described and compared on the basis of observation and measurements under real working conditions, laboratory testing, and computer simulations. The main conclusions are drawn as follows:

(1) The asynchronous movement of the contact wire and the messenger wire causes the horizontal vibration of the droppers under real working conditions.

(2) The horizontal vibration behaviour of a dropper under real working conditions can be reproduced in the laboratory using a spring connected on the upper end of the dropper and an active movement driving on the lower end of the dropper.

(3) To investigate the horizontal vibration behaviour after the large loosening of the droppers, the dynamic simulation model can be built through several sections, where each section is considered a rigid body, and the sections are connected with planar torsion springs. The coefficient of association between the virtual sensor load in the simulation and the real sensor load in the laboratory test is 0.9578 . 
Thus, a simulation method of droppers considering horizontal vibration behaviour was proposed. The proposed method provided an increasingly accurate means of simulating the load of the droppers under real working conditions. However, the influence of horizontal vibration must be explored. The study of the relationships between the dynamic internal load of a dropper and its fatigue life may be meaningful in dropper fatigue life prediction.

\section{ACKNOWLEDGEMENT}

This work was supported by the National Natural Science Foundation of China (NSFC; Grant number U1534209).

\section{REFERENCES}

[1] Wu, J. (2018). Pantograph and Contact Line System, Academic Press, London

[2] Liu, X.-Y.; Peng, J.-F.; Tan, D.-Q.; Xu, Z.-B.; Liu, J.-H.; Mo, J.-L.; Zhu, M.-H. (2018). Failure analysis and optimization of integral droppers used in high speed railway catenary system, Engineering Failure Analysis, Vol. 91, 496-506, doi:10.1016/j.engfailanal.2018.02.003

[3] Lee, Y.-L.; Tjhung, T. (2012). Rainflow cycle counting techniques, Lee, Y.-L.; Barkey, M. E.; Kang, H.-T. (Eds.), Metal Fatigue Analysis Handbook, Butterworth-Heinemann, Waltham, 89114

[4] Qi, G.; Zhao, H.; Xiao, X.; Xu, H.; Li, H. (2018). Numerical simulation of dynamic stress and analysis of fatigue load characteristics for high-speed railway catenary droppers, China Mechanical Engineering, Vol. 29, No. 9, 1063-1068, doi:10.3969/j.issn.1004-132X.2018.09.009

[5] Chen, L.; Peng, P.; He, F. (2018). Fatigue life analysis of dropper used in pantograph-catenary system of high-speed railway, Advances in Mechanical Engineering, Vol. 10, No. 5, 10 pages, doi: $10.1177 / 1687814018776135$

[6] Yang, G. (2017). Discussion of a life prediction method for catenary parts, Railway Quality Control, Vol. 45, No. 9, 30-33, doi:10.3969/j.issn.1006-9178.2017.09.010

[7] Peng, P.; Chen, L.; Wang, W.; He, F. (2019). The effect of frequency and amplitude of dropper on its fatigue life, Journal of Railway Science and Engineering, Vol. 2019, No. 2, 471-477, doi:10.19713/j.cnki.43-1423/u.2019.02.025

[8] Liu, J.; Zhang, J.; Chu, W.; Liu, Z. (2019). Analysis of mechanical properties of key parts and components of high-speed railway catenary, Electric Railway, Vol. 2019, No. 3, 38-42, doi:10.19587/j.cnki.1007-936x.2019.03.010

[9] Yang, G. (2016). Failure analysis and improvement effect verification of droppers in high-speed railway catenary, Railway Quality Control, Vol. 44, No. 9, 21-23, doi:10.3969/j.issn.10069178.2016.09.008

[10] Lacombe, A.; Landon, Y.; Paredes, M.; Chirol, C.; Benaben, A. (2019). Numerical simulation of the reaming process in aluminium 2024-T351-analysis of the evolution of the material stress state, DYNA Ingenieria e Industria, Vol. 94, No. 1, Paper 02050058, 10 pages, doi:10.6036/8900

[11] Tan, D.; Mo, J.; Peng, J.; Luo, J.; Chen, W.; Zhu, M. (2018). Research and prospect on highspeed catenary component failure, Journal of Southwest Jiaotong University, Vol. 53, No. 3, 610619, doi:10.3969/j.issn.0258-2724.2018.03.024

[12] Jiang, X.; Gu, X.; Deng, H.; Zhang, Q.; Mo, J. (2019). Research on damage mechanism and optimization of integral dropper string based on fretting theory, Journal of the China Railway Society, Vol. 41, No. 6, 40-45, doi:10.3969/j.issn.1001-8360.2019.06.006

[13] Rodriguez Ramirez, J. A.; de Jesus Mariaca Beltran, Y.; Garcia Salmoran, I. A.; Clemente Mirafuente, C. M.; Rodriguez Ramirez, J. A.; Acosta-Flores, M.; Garcia Castrejon, J. C. (2019). New methodology for the analysis of mechanical system using scale models and similarity laws, DYNA Ingenieria e Industria, Vol. 94, No. 1, 59-66, doi:10.6036/8825

[14] Ramadani, R.; Belsak, A.; Kegl, M.; Predan, J.; Pehan, S. (2018). Topology optimization based design of lightweight and low vibration gear bodies, International Journal of Simulation Modelling, Vol. 17, No. 1, 92-104, doi:10.2507/ijsimm17(1)419 
[15] Guan, J.; Wu, J. (2017). Building and confirmation for dynamic simulation model of pantograph and catenary, Journal of Railway Science and Engineering, Vol. 14, No. 11, 2444-2451, doi: $\frac{10.3969 / j . i s s n .1672-7029.2017 .11 .023}{2}$

[16] Zhao, H.; Xiao, X.; Qi, G.; Xu, H.; Li, H. (2019). Analysis of fatigue life of catenary dropper for high-speed railway, Engineering Journal of Wuhan University, Vol. 2019, No. 4, 351-357, doi:10.14188/j.1671-8844.2019-04-011

[17] Benet, J.; Cuartero, N.; Cuartero, F.; Rojo, T.; Tendero, P.; Arias, E. (2013). An advanced 3Dmodel for the study and simulation of the pantograph catenary system, Transportation Research Part C: Emerging Technologies, Vol. 36, 138-156, doi:10.1016/j.trc.2013.08.004

[18] Pombo, J.; Ambrósio, J. (2013). Environmental and track perturbations on multiple pantograph interaction with catenaries in high-speed trains, Computers \& Structures, Vol. 124, 88-101, doi:10.1016/j.compstruc.2013.01.015

[19] Gregori, S.; Tur, M.; Nadal, E.; Fuenmayor, F. J. (2017). An approach to geometric optimisation of railway catenaries, Vehicle System Dynamics - International Journal of Vehicle Mechanics and Mobility, Vol. 56, No. 8, 1162-1186, doi:10.1080/00423114.2017.1407434

[20] Massat, J.-P.; Balmes, E.; Bianchi, J.-P.; van Kalsbeek, G. (2015). OSCAR statement of methods, Vehicle System Dynamics - International Journal of Vehicle Mechanics and Mobility, Vol. 53, No. 3, 370-379, doi:10.1080/00423114.2015.1005016

[21] Cho, Y. H. (2008). Numerical simulation of the dynamic responses of railway overhead contact lines to a moving pantograph, considering a nonlinear dropper, Journal of Sound and Vibration, Vol. 315, No. 3, 433-454, doi:10.1016/j.jsv.2008.02.024

[22] Bruni, S.; Ambrosio, J.; Carnicero, A.; Cho, Y. H.; Finner, L.; Ikeda, M.; Kwon, S. Y.; Massat, J.-P.; Stichel, S.; Tur, M.; Zhang, W. (2015). The results of the pantograph-catenary interaction benchmark, Vehicle System Dynamics - International Journal of Vehicle Mechanics and Mobility, Vol. 53, No. 3, 412-435, doi:10.1080/00423114.2014.953183

[23] Vo Van, O.; Massat, J.-P.; Balmes, E. (2017). Waves, modes and properties with a major impact on dynamic pantograph-catenary interaction, Journal of Sound and Vibration, Vol. 402, 51-69, doi:10.1016/j.jsv.2017.05.008

[24] Gregori, S.; Tur, M.; Nadal, E.; Aguado, J. V.; Fuenmayor, F. J.; Chinesta, F. (2017). Fast simulation of the pantograph-catenary dynamic interaction, Finite Elements in Analysis and Design, Vol. 129, 1-13, doi:10.1016/j.finel.2017.01.007

[25] Trzepiecinski, T.; Lemu, H. G.; Fejkiel, R. (2017). Numerical simulation of effect of friction directionality on forming of anisotropic sheets, International Journal of Simulation Modelling, Vol. 16, No. 4, 590-602, doi:10.2507/ijsimm16(4)3.392

[26] Rui, X.; He, B.; Lu, Y.; Lu, W.; Wang, G. (2005). Discrete time transfer matrix method for multibody system dynamics, Multibody System Dynamics, Vol. 14, No. 3-4, 317-344, doi:10.1007/s11044-005-5006-1 